\title{
On the Endogenous Formation of Energy Efficient Cooperative Wireless Networks
}

\author{
Fatemeh Fazel and D. Richard Brown III \\ $\{$ fazel,drb\}@ece.wpi.edu \\ Electrical and Computer Engineering Department \\ Worcester Polytechnic Institute \\ Worcester, MA 01609
}

\begin{abstract}
In wireless networks, it is well-known that intermediate nodes can be used as cooperative relays to reduce the transmission energy required to reliably deliver a message to an intended destination. When the network is under a central authority, energy allocations and cooperative pairings can be assigned to optimize the overall energy efficiency of the network. In networks with autonomous selfish nodes, however, nodes may not be willing to expend energy to relay messages for others. This problem has been previously addressed through the development of extrinsic incentive mechanisms, e.g. virtual currency, or the insertion of altruistic nodes in the network to enforce cooperative behavior. This paper considers the problem of how selfish nodes can decide on an efficient energy allocation and endogenously form cooperative partnerships in wireless networks without extrinsic incentive mechanisms or altruistic nodes. Using tools from both cooperative and non-cooperative game theory, the three main contributions of this paper are (i) the development of Pareto-efficient cooperative energy allocations that can be agreed upon by selfish nodes, based on axiomatic bargaining techniques, (ii) the development of necessary and sufficient conditions under which "natural" cooperation is possible in systems with fading and non-fading channels without extrinsic incentive mechanisms or altruistic nodes, and (iii) the development of techniques to endogenously form cooperative partnerships without central control. Numerical results with orthogonal amplify-and-forward (OAF) cooperation are also provided to quantify the energy efficiency of a wireless network with sources selfishly allocating transmission/relaying energy and endogenously forming cooperative partnerships with respect to a network with centrally optimized energy allocations and pairing assignments.
\end{abstract}

\section{INTRODUCTION}

Multihop or cooperative transmission is often used in wireless ad hoc networks to increase energy efficiency by allowing packets to be delivered over several short links [1]. One or more intermediate nodes between the source and destination can assist in the transmission by forwarding or relaying the packet along the route to the destination. Autonomous nodes acting in their own self-interest, however, may refuse to use their limited resources to forward packets for other nodes. This can lead to inefficient use of the network resources since messages may have to be retransmitted or re-routed through different paths to the destination node [2].

Several techniques have been proposed to encourage cooperation and improve the efficiency of wireless ad hoc networks with selfish autonomous nodes. A comprehensive study of these techniques can be found in [3]. One well-studied technique to encourage cooperation among selfish nodes is the development of extrinsic incentive mechanisms, e.g. virtual currency [4], [5], where nodes are reimbursed for cooperation. The idea of virtual currency is intuitively appealing in many scenarios, but the use of virtual currency has the potential for fraud and/or collusion as discussed in [6]. Another technique that can induce cooperation is the introduction of altruistic nodes into the network [7] that punish misbehaving nodes. While both of these techniques have been shown to encourage cooperation among selfish nodes, they both require some level of central authority in the network to perform accounting or to strategically insert altruistic nodes. They also implicitly assume that the near-term costs and benefits of cooperative behavior are one-sided, hence remuneration is necessary to enable cooperation. While this assumption is true in some cases, recent studies, e.g. [8], have shown that the benefits of cooperation can be two-sided and have considered the question of when "natural" cooperation is possible in large networks without any central authority. In [9], a two-player relaying game based on the orthogonal amplify-and-forward (OAF) cooperative transmission protocol [10] was analyzed in a noncooperative game-theoretic framework and it was shown that natural cooperation without extrinsic incentive mechanisms or altruistic nodes can emerge under certain conditions on the channels. Two limitations of this work, however, are that it used centrally-controlled energy allocations and did not consider networks with more than two source nodes.

This paper considers the problem of how selfish nodes can locally decide on an efficient energy allocation and endogenously form cooperative partnerships in wireless networks with two or more source nodes and without any sort of extrinsic incentive mechanisms, altruistic nodes, or central authority. We first use bargaining tools from cooperative game theory to determine efficient energy allocations that can be locally computed and agreed to by a pair of selfish nodes. We then develop a repeated-game framework and employ tools from non-cooperative game theory to describe necessary and sufficient conditions under which natural cooperation between a pair of nodes is possible. To extend our results to networks with more than two source nodes, we then consider the question of how to endogenously form cooperative partnerships in general networks and propose the use of the "stable 
roommates" algorithm [11], [12] to form partnerships that are stable with respect to unilateral or bilateral deviations. Finally, numerical results with orthogonal amplify-and-forward (OAF) cooperation are provided to quantify the energy efficiency of a wireless network with sources selfishly allocating transmission/relaying energy and endogenously forming cooperative partnerships with respect to a network with centrally optimized energy allocations and pairing assignments.

Unlike the previous studies on this subject, the novelty of the approach in this paper is that the nodes in the network behave selfishly without any form of central authority, community enforcement, or extrinsic incentive mechanisms. Selfish autonomous nodes endogenously form cooperative partnerships, locally determine efficient energy allocations, and cooperate by relaying messages during transmission sessions with multiple frames. Throughout this paper, we assume that nodes exhibit rational individual choice behavior, meaning that each individual source node has a consistent preference relation over all possible energy allocations and partners, and always chooses the most preferred feasible alternative. We also assume that nodes can always refuse to cooperate if it is in their best interest to do so.

The rest of the paper is organized as follows. Section II introduces the system model used throughout the paper. In Section III we present a two-player relaying game in stagegame formulation, develop axiomatic bargaining solutions to determine an optimum energy allocation that two selfish players can agree upon, and then develop necessary and sufficient conditions under which selfish nodes will cooperate and not defect under a repeated-game formulation for both fading and non-fading channels. Section V extends these twoplayer results to networks with $K>2$ sources and describes a technique in which the sources can endogenously form stable cooperative pairings. Section VI provides numerical energy efficiency examples based on OAF cooperative transmission and concluding remarks are made in Section VII.

The rest of the paper is organized as follows. Section II introduces the system model used throughout the paper. In Section III we present a two-player relaying game in stage game and repeated game formulation for both fading and nonfading channel conditions and Section IV provides several axiomatic bargaining solutions to determine an optimum energy allocation that the two selfish players can agree upon. Section V extends the two-player game to more than two players by allowing the transmitting sources to form stable cooperative pairs. Section VI provides a numerical example based on OAF cooperative relaying. Concluding remarks are made in Section VII.

\section{SySTEM MOdEL}

We consider an ad hoc wireless network with $L$ half-duplex nodes and a discrete model of time where nodes transmit information to other nodes in the network in transmission sessions of variable duration. The sets of source nodes and destination nodes in a given transmission session are denoted as $\mathcal{S}$ and $\mathcal{D}$, respectively, where $|\mathcal{S}|=|\mathcal{D}|=K \leq L / 2$ and
$\mathcal{S} \cap \mathcal{D}=\emptyset$. The destination node for source node $i \in \mathcal{S}$ is denoted as $d_{i} \in \mathcal{D}$. It is assumed that the number of nodes and/or the amount of offered network traffic is sufficiently large such that, in any given transmission session, $K \geq 2$ source nodes wish to transmit independent information to distinct destination nodes in the network.

In each transmission session, the $K$ source nodes involved in the transmission session take turns transmitting using timedivision multiple access (TDMA). A transmission session is composed of $N \geq 1$ frames and each frame is composed of $2 K$ timeslots as shown in Figure 1 for the case when $K=2$. The channel $h_{i j}[n]$ between node $i \in \mathcal{S}$ and node $j \in \mathcal{D} \cup$ $\mathcal{S} \backslash i$ in frame $n$ is assumed to be frequency non-selective. The squared channel magnitude between node $i$ and $j$ in frame $n$, normalized with respect to the power of the additive white Gaussian noise (AWGN) in the channel, is denoted as $H_{i j}[n]$.

In the first $K$ timeslots of each frame, each source node transmits a packet to its destination. Due to the undirected nature of wireless transmission, the TDMA transmissions in the first $K$ timeslots are also overheard by the other source nodes in the transmission session. The signal received by node $j$ from node $i$ in frame $n$ is given as

$$
y_{i j}[n]=\sqrt{H_{i j}[n]} x_{i}[n]+u_{i j}[n] .
$$

for all $i \in \mathcal{S}$ and all $j \in \mathcal{D} \cup \mathcal{S} \backslash i$ in the current transmission session where $x_{i}[n]$ is the packet transmitted by source node $i$ in frame $n$ and $u_{i j}[n]$ is zero-mean unit-variance AWGN. In the remaining $K$ timeslots of the frame, each source node can potentially help one other source node by relaying a packet to its intended destination. When source node $j \in \mathcal{S} \backslash i$ elects to relay a packet for source node $i$, it transmits a function of the observation received from source node $i$ in the first half of the frame. Destination node $d_{i} \in \mathcal{D}$ receives

$$
z_{j d_{i}}[n]=\sqrt{H_{j d_{i}}[n]} f\left(y_{i j}[n]\right)+v_{j d_{i}}[n]
$$

where the relaying function $f$ depends on the cooperative protocol and $v_{j d_{i}}[n]$ is zero-mean unit-variance AWGN. All noise terms are assumed to be spatially and temporally white. Note that each destination $d_{i} \in \mathcal{D}$ always receives at least one observation in each frame, i.e. the direct transmission $y_{i d_{i}}[n]$, and may receive two observations if another source node elects to relay the packet from source node $i$.

It is assumed that the normalized channel magnitudes $\boldsymbol{H}[n]=\left\{H_{i j}[n]\right\}$ are quasi-static and fading in the sense that $\boldsymbol{H}[n]$ is constant over the duration of each frame, but are independent and identically distributed (i.i.d.) in different frames of the transmission session. The current channel state is assumed to be known by the $K$ source nodes involved in the current transmission session. Channel phases are only assumed to be known at the respective receivers.

\section{Two-Player Relaying Game}

This section considers the scenario when there are two source nodes, denoted as $\mathcal{S}=\{1,2\}$, that wish to communicate independent information to two distinct destination nodes, 


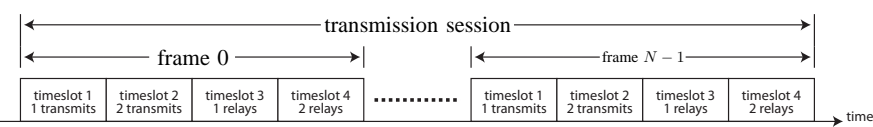

Fig. 1. A transmission session composed of $N$ frames with $\mathcal{S}=\{1,2\}$.

denoted as $\mathcal{D}=\{3,4\}$. We extend the ideas developed in this section to the case with $K>2$ source nodes in Section V.

\section{A. Stage Game Formulation}

A stage game is defined in terms of the players, available actions, and payoffs received by each player as a consequence of the actions for one frame of the current transmission session. The players in the game are the source nodes. In the first two timeslots of frame $n$, each source node transmits to its destination using transmit energy $\mathcal{E}_{1}[n]$ and $\mathcal{E}_{2}[n]$, respectively. If a source node does not request relaying from the other source node, i.e. it uses direct transmission to its destination, it will transmit with sufficient energy to satisfy a minimum quality-of-service (QoS) constraint, e.g. SNR or rate, at its destination based on the current channel state. For example, if the required signal-to-noise ratio at destination node $d_{i}$ is $\rho$, then source node $i$ will transmit with energy $\mathcal{E}_{i}[n]=\rho / H_{i d_{i}}[n]$ in frame $n$ when it uses direct transmission. We denote the required direct transmission energy for source node $i$ in frame $n$ as $\mathcal{E}_{i}^{d t}[n]$. If a source node requests relaying from the other source node in frame $n$, it will transmit with energy $0<\mathcal{E}_{i}[n]<\mathcal{E}_{i}^{d t}[n]$.

Referring to the timeslot schedule in Figure 1, if node 2 has requested relaying, then node 1 must decide in timeslot 3 whether to fulfill this relaying request. Since both source nodes know the channel state, node 1 can determine the minimum relaying energy $\mathcal{E}_{1}^{r_{\text {min }}}[n]$ required to ensure the QoS constraint is satisfied at destination node 4 [13]. Although node 1 can choose any non-negative relaying energy $\mathcal{E}_{1}^{r}[n]$, it will never rationally choose a relaying energy larger than the minimum required relaying energy since there is no benefit to either source node if a node expends excess relaying energy. If source node 1 transmits with relaying energy less than the minimum required relaying energy, then the packet will not be received at destination node 4 with the required QoS and node 2 will need to transmit with additional energy at the end of the frame to ensure the QoS constraint is satisfied. For these reasons, we assume that node 1 chooses from the discrete set of actions "do not relay" $\left(a_{1}[n]=\mathrm{DNR} \Leftrightarrow \mathcal{E}_{1}^{r}[n]=0\right)$ and "relay with minimum required relaying energy" $\left(a_{1}[n]=\mathrm{R} \Leftrightarrow \mathcal{E}_{1}^{r}[n]=\right.$ $\left.\mathcal{E}_{1}^{r_{\text {min }}}[n]\right)$ in frame $n$. If source node 1 chooses the action DNR when $\mathcal{E}_{1}^{r_{\text {min }}}[n]>0$, then node 2 will transmit at the end of the frame with the remaining energy required to ensure the QoS constraint is satisfied at node 4 . Since the channel magnitudes are assumed to be constant over the duration of the frame, the total transmission energy expended by node 2 in this case will be the same as if node 2 had used direct transmission in timeslot 2 . If source node 1 chooses the action $R$, the packet will be received by node 4 at the required QoS level without any additional transmission energy from node 2 .
In timeslot 4 , if node 1 has requested relaying, node 2 must also decide between the actions DNR and R. The situation is the same in this case as when node 1 relays for node 2 except that node 2 has the advantage of having just observed whether or not node 1 fulfilled its relaying request and can choose its action accordingly.

Since packets from both source nodes are always delivered to each destination irrespective of whether relaying requests are fulfilled or not, we define the stage-game payoff as the transmission energy saved in the current frame with respect to direct transmission. The payoff received by source node $i$ in frame $n$ for actions

$\boldsymbol{a}[n]=\left(a_{1}[n], a_{2}[n]\right) \in\{(\mathrm{DNR}, \mathrm{DNR}),(\mathrm{DNR}, \mathrm{R}),(\mathrm{R}, \mathrm{DNR}),(\mathrm{R}, \mathrm{R})\}$

is denoted as $\pi_{i}(\boldsymbol{a}[n], n)$. Note that whenever $\boldsymbol{a}[n]=$ (DNR, DNR), both source nodes receive a payoff of zero. If source node $i$ chooses $\mathrm{R}$ and node $j$ chooses DNR, then node $i$ receives a payoff of $\pi_{i}(\boldsymbol{a}[n], n)=-\mathcal{E}_{i}^{r_{\text {min }}}$ and node $j$ receives a payoff of $\pi_{j}(\boldsymbol{a}[n], n)=\mathcal{E}_{j}^{*}$ where

$$
\mathcal{E}_{j}^{*}[n]:=\mathcal{E}_{j}^{d t}[n]-\mathcal{E}_{j}[n]>0
$$

is defined as the energy saved by node $j$ with respect to direct transmission if node $j$ requests relaying and node $i \neq j$ fulfills the relaying request by relaying with sufficient energy to ensure the QoS constraint is satisfied at destination node $d_{j}$. Finally, if $\boldsymbol{a}[n]=(\mathrm{R}, \mathrm{R})$, the source nodes receive payoffs $\left(\pi_{1}(\boldsymbol{a}[n], n), \pi_{2}(\boldsymbol{a}[n], n)\right)=\left(\mathcal{E}_{1}^{*}-\mathcal{E}_{1}^{r_{\text {min }}}, \mathcal{E}_{2}^{*}-\mathcal{E}_{2}^{r_{\text {min }}}\right)$. Figure 2 summarizes the two-player relaying game in extensive form [14] and shows the payoffs received by each source node as a function of the actions chosen by the players in the current frame.

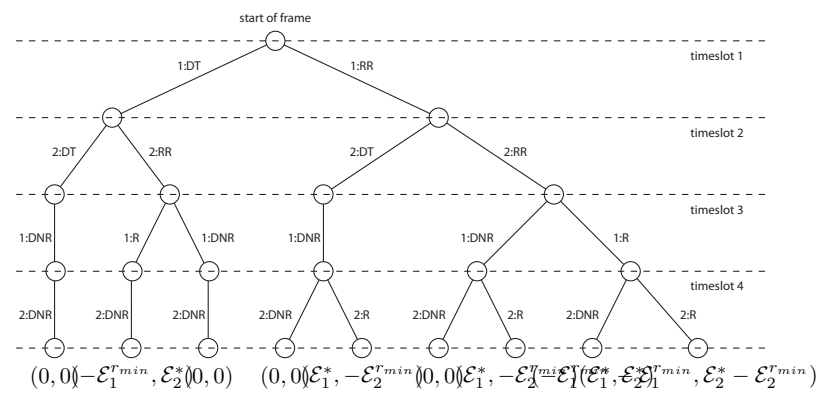

Fig. 2. Two-player relaying game in extensive form with source nodes $\mathcal{S}=$ $\{1,2\}$. The actions DT, RR, R, and DNR correspond to "direct transmission (no relay request)", "request relay", "relay", and "do not relay", respectively. The pairs at the bottom of the tree correspond to the payoffs of nodes 1 and 2 , respectively, at the end of the frame.

1) Stage Game Analysis: Inspection of the payoff pairs in Figure 2 shows that, when node 2 is requested to relay such that $\mathcal{E}_{2}^{r_{\text {min }}}>0$, node 2 will choose the action DNR because its payoff of choosing DNR is always better than choosing $\mathrm{R}$, irrespective of node 1's actions. Knowing this, node 1 will also choose the action DNR for the same reasons. Since both source nodes know that any relay requests will always be rejected, both source nodes will choose to communicate with their respective destinations by direct transmission and receive 
the payoff pair $\left(\pi_{1}(\boldsymbol{a}[n], n), \pi_{2}(\boldsymbol{a}[n], n)\right)=(0,0)$.

To formalize this result, we briefly review the concept of a Nash Equilibrium (NE) [14]. In a $k$-player game, the action profile $\left(a_{1}^{*}, \ldots, a_{k}^{*}\right)$ is an NE if, for each player $i, a_{i}^{*}$ is player $i$ 's best response to $\boldsymbol{a}_{-i}^{*}$, where $\boldsymbol{a}_{-i}$ denotes the actions of all the players except player $i$. In frame $n$, this can be expressed as

$$
\pi_{i}\left(\left\{a_{i}^{*}[n], \boldsymbol{a}_{-i}^{*}[n]\right\}, n\right) \geq \pi_{i}\left(\left\{a_{i}[n], \boldsymbol{a}_{-i}^{*}[n]\right\}, n\right)
$$

for all $a_{i}$ in the set of available actions for player $i$ and where $\pi_{i}$ is the payoff function player $i$. Intuitively, if all of the players are choosing NE actions, no player can increase their payoff by unilaterally deviating from the NE action profile. It is not difficult to show that the only NE of the two-player relaying stage game is $\boldsymbol{a}[n]=(\mathrm{DNR}, \mathrm{DNR})$.

The dilemma in this result is that both nodes could potentially receive a payoff better than $(0,0)$ by accepting relay requests when $\mathcal{E}_{1}^{*}-\mathcal{E}_{1}^{r_{\text {min }}}>0$ and $\mathcal{E}_{2}^{*}-\mathcal{E}_{2}^{r_{\text {min }}}>0$. In other words, if the channel state is such that both nodes could save energy through mutual cooperation, both nodes would do better by choosing $\boldsymbol{a}[n]=(\mathrm{R}, \mathrm{R})$ than $\boldsymbol{a}[n]=(\mathrm{DNR}, \mathrm{DNR})$. Nevertheless, analysis of the stage game yields only one NE action profile for selfish players: mutual non-cooperation. In the following section, we extend this stage-game analysis to a repeated game formulation and show that, unlike a single-stage game, a repeated game with uncertain ending can include a mutually cooperative NE for selfish players.

\section{B. Repeated Game Formulation}

Since each transmission session is composed of $N \geq 1$ frames, the stage game formulation developed in the prior section can be extended to a repeated game model where the players interact over multiple stage games. If $N$ is known to both source nodes in the current transmission session, backward induction arguments can be used to show that both players will choose $\boldsymbol{a}[n]=$ (DNR, DNR) in each stage game. To see this, first consider the last stage game. Since there is no possibility of gain from future cooperation, node 2 will rationally choose DNR to maximize its payoff. Knowing this, node 1 will also choose DNR for the same reasons. Since each node knows that the other node will choose DNR in the last stage game, they will also choose $\boldsymbol{a}[n]=(\mathrm{DNR}, \mathrm{DNR})$ in the second to last stage game, and so on, ensuring that the only rational strategy for both source nodes is to reject relay requests in all of the stage games [15, p.10].

Now consider the scenario when the number of frames in the current transmission session is not known by the source nodes. Specifically, we consider the case when the transmission session continues after the current frame with fixed probability $\delta$, where $\delta$ is known to both source nodes. In this case, the number of frames $N$ is a geometrically distributed random variable with probability mass function $p_{N}(n)=(1-\delta) \delta^{n-1}$ for $n=1,2, \ldots$. Since the number of frames in the current transmission session is not known to the source nodes (and, as will be discussed in Section III-B1, the payoffs in future frames may also be unknown), both source nodes seek to maximize their expected total payoff in the transmission session. We define the expected total payoff of node $i$ as

$$
\Pi_{i}:=\mathrm{E}\left\{\sum_{n=0}^{N-1} \pi_{i}(\boldsymbol{a}[n], n)\right\}
$$

where $N$ is random and $\pi_{i}(\boldsymbol{a}[n], n)$ may be random. Under the assumption that the stage-game payoffs are independent of $N$, the expected total payoff can be shown to be equivalent to a repeated game having an infinite number of stages with future payoffs discounted according to the expected duration of the game [15]. For player $i$, this can be expressed as

$$
\Pi_{i}=\sum_{n=0}^{\infty} \delta^{n} \mathrm{E}\left\{\pi_{i}(\boldsymbol{a}[n], n)\right\}
$$

where $\delta$ is called the discount factor and $\pi_{i}(\boldsymbol{a}[n], n)$ is the $i^{\text {th }}$ player's payoff in frame $n$ given action profile $\boldsymbol{a}[n]$.

In repeated games, players use a strategy to specify their actions in each stage game as a function of the channel state, cooperative protocol, QoS constraint, and previous actions of the other players. We define a TRIGGER strategy in the repeated two-player relaying game as follows: if $\mathcal{E}_{i}^{r_{\text {min }}}[n]>0$, player $i$ chooses the action $a_{i}[n]=\mathrm{R}$ unless the other player has previously chosen DNR when relaying was requested. If player $i$ chooses DNR when $\mathcal{E}_{i}^{r_{\text {min }}}[n]>0$, then player $i$ is said to defect. If either player defects, the other player "triggers" punishment by choosing DNR in all future stage games (note that, since node 2 chooses its action after it observes the action of node 1 in the current stage game, node 2 will trigger punishment by playing DNR in the current stage game).

1) Repeated Game Analysis: In our analysis of the repeated game scenario, the channel states in the current and previous frames are assumed to be known to both sources. The channel states in future frames are not known; only their distribution is known. The energy allocation $\mathcal{E}[n]=$ $\left\{\mathcal{E}_{1}[n], \mathcal{E}_{2}[n], \mathcal{E}_{1}^{r_{\text {min }}}[n], \mathcal{E}_{2}^{r{ }^{\text {min }}}[n]\right\}$ is assumed to dynamically determined in each frame $n=0,1, \ldots$ according to the known channel state, the cooperative protocol, and the QoS constraint. The following proposition establishes necessary and sufficient conditions under which the (TRIGGER,TRIGGER) strategy profile is an $\mathrm{NE}$ of the repeated two-player relaying game with uncertain ending in systems with quasi-static i.i.d. fading channels.

Proposition 1. In a system with quasi-static i.i.d. fading channels, the strategy profile (TRIGGER, TRIGGER) is an NE of the repeated two-player relaying game with uncertain ending if and only if $\mathcal{E}_{1}^{r_{\text {min }}}[n] \leq \mathcal{E}_{1}^{*}[n]+\frac{\delta}{1-\delta} \overline{\mathcal{E}}_{1}$ and $\mathcal{E}_{2}^{r_{\text {min }}}[n] \leq \frac{\delta}{1-\delta} \overline{\mathcal{E}}_{2}$ for all $n=0,1, \ldots$ where $\overline{\mathcal{E}}_{i}:=E\left\{\mathcal{E}_{i}^{*}[n]-\mathcal{E}_{i}^{r_{\text {min }}}[n]\right\}$.

Proof: In frame $n^{\prime}$, if both nodes have faithfully played and continue to play the strategy profile (TRIGGER,TRIGGER), 
they will receive an expected total payoff of

$$
\begin{aligned}
\Pi_{i}= & \sum_{n=0}^{n^{\prime}-1} \delta^{n}\left(\mathcal{E}_{i}^{*}[n]-\mathcal{E}_{i}^{r_{\min }}[n]\right) \\
+ & \delta^{n^{\prime}}\left(\mathcal{E}_{i}^{*}\left[n^{\prime}\right]-\mathcal{E}_{i}^{r_{\min }}\left[n^{\prime}\right]\right)+\sum_{n=n^{\prime}+1}^{\infty} \delta^{n} \overline{\mathcal{E}}_{i} .
\end{aligned}
$$

The first and second terms in this expression correspond to the known total payoff of the previous frames and the known payoff of the current frame, respectively. The final term in this expression corresponds to the expected total payoff from mutual cooperation in future stage games where the i.i.d. channel state assumption has been used to remove the dependence of the mean on $n$.

If node 1 deviates from the TRIGGER strategy by defecting in stage game $n^{\prime}$, it will receive a total payoff of

$$
\Pi_{1}=\sum_{n=0}^{n^{\prime}-1} \delta^{n}\left(\mathcal{E}_{1}^{*}[n]-\mathcal{E}_{1}^{r_{\text {min }}}[n]\right)
$$

because node 2 will punish node 1 immediately for its defection in the current stage game. Note that this total expected payoff does not exceed the total expected payoff from faithfully playing the TRIGGER strategy when $\delta^{n^{\prime}}\left(\mathcal{E}_{1}^{*}\left[n^{\prime}\right]-\right.$ $\left.\mathcal{E}_{1}^{r_{\text {min }}}\left[n^{\prime}\right]\right)+\sum_{n=n^{\prime}+1}^{\infty} \delta^{n} \overline{\mathcal{E}}_{1} \geq 0$. Hence, node 1 has no incentive to deviate from the strategy profile (TRIGGER,TRIGGER) when $\mathcal{E}_{1}^{r_{\min }}\left[n^{\prime}\right] \leq \mathcal{E}_{1}^{*}\left[n^{\prime}\right]+\frac{\delta}{1-\delta} \overline{\mathcal{E}}_{1}$.

If node 2 deviates from the TRIGGER strategy by defecting in stage game $n^{\prime}$, it is punished by node 1 in the next stage game and receives a total expected payoff of

$$
\Pi_{2}=\sum_{n=0}^{n^{\prime}-1} \delta^{n}\left(\mathcal{E}_{2}^{*}[n]-\mathcal{E}_{2}^{r_{\min }}[n]\right)+\delta^{n^{\prime}} \mathcal{E}_{2}^{*}\left[n^{\prime}\right] .
$$

The second term here corresponds to the payoff received by node 2 in stage game $n^{\prime}$ when its packet is forwarded by node 1 but it does not reciprocate. This total expected payoff does not exceed the total expected payoff from faithfully playing the TRIGGER strategy when $\mathcal{E}_{2}^{r_{\text {min }}}\left[n^{\prime}\right] \leq \frac{\delta}{1-\delta} \overline{\mathcal{E}}_{2}$.

Proposition 1 implies that, as long as both sources can find an energy allocation such that they are not requested to expend "too much" relaying energy in the current frame, then mutual cooperation (with the threat of punishment for defection) is an $\mathrm{NE}$ of the repeated two-player relaying game with uncertain ending. In other words, both source nodes have no incentive to defect when they can expect to receive more long-term benefit from cooperation than short-term benefit from defection. Note that the strategy profile (ALWAYS DEFECT, ALWAYS DEFECT) is also an NE of the repeated two-player relaying game with uncertain ending since neither player stands to gain from cooperation with an opponent that always defects.

As a special case of Proposition 1, we can also consider a system with non-fading channels where the channel state is the same over all of the frames in the transmission session, i.e. $\boldsymbol{H}[n] \equiv\left\{H_{12}, H_{13}, H_{14}, H_{21}, H_{23}, H_{24}\right\}$ for all $n=0,1, \ldots$, and the sources use fixed energy allocations, i.e. $\mathcal{E}[n] \equiv\left\{\mathcal{E}_{1}, \mathcal{E}_{2}, \mathcal{E}_{1}^{r_{\text {min }}}, \mathcal{E}_{2}^{r_{\text {min }}}\right\}$ for all $n=0,1, \ldots$ The following lemma establishes necessary and sufficient conditions under which the (TRIGGER,TRIGGER) strategy profile is an NE of the repeated two-player relaying game with uncertain ending in systems with non-fading channels.

Lemma 1. In a system with non-fading channels, the strategy profile (TRIGGER,TRIGGER) is an NE of the repeated twoplayer relaying game with uncertain ending if and only if $\mathcal{E}_{1}^{r_{\text {min }}} \leq \mathcal{E}_{1}^{*}$ and $\mathcal{E}_{2}^{r_{\text {min }}} \leq \delta \mathcal{E}_{2}^{*}$.

The proof of Lemma 1 follows from Proposition 1 by substituting $\overline{\mathcal{E}}_{i}=\mathcal{E}_{i}^{*}-\mathcal{E}_{i}^{r_{\text {min }}}$ for $i \in\{1,2\}$. Unlike the case with fading channels, all of the future payoffs are known when the channels are non-fading; only the duration of the transmission session is unknown. Both source nodes have no incentive to defect when they expect to receive more longterm benefit from cooperation than short-term benefit from defection.

In each frame $n=0,1, \ldots$, Proposition 1 and Lemma 1 identify a set of feasible energy allocations under which selfish nodes will rationally choose mutual cooperation. This set might be empty, depending on the channel state, cooperative protocol, and QoS constraint, in which case the only NE is for both sources to use direct transmission. When this set is not empty, there remains the question of how much relaying energy the sources should demand of each other. The initial transmit energies $0<\mathcal{E}_{1}[n] \leq \mathcal{E}_{1}^{d t}[n]$ and $0<\mathcal{E}_{2}[n] \leq \mathcal{E}_{2}^{d t}[n]$, when combined with the channel state $\boldsymbol{H}[n]$, the cooperative protocol, and the QoS constraint, imply the minimum required relaying energies $\mathcal{E}_{1}^{r_{\text {min }}}[n]$ and $\mathcal{E}_{2}^{r_{\text {min }}}[n]$. Since the channel state is known to both source nodes, one possible strategy would be for both source nodes to choose $\mathcal{E}_{1}[n]$ and $\mathcal{E}_{2}[n]$ such that they request the maximum relaying energy from the other source under the conditions of Proposition 1, i.e. node 2 selects $\mathcal{E}_{2}[n]$ such that $\mathcal{E}_{1}^{r_{\text {min }}}[n]=\mathcal{E}_{1}^{*}[n]+\frac{\delta}{1-\delta} \overline{\mathcal{E}}_{1}$ and node 1 selects $\mathcal{E}_{1}[n]$ such that $\mathcal{E}_{2}^{r_{\text {min }}}[n]=\frac{\delta}{1-\delta} \overline{\mathcal{E}}_{2}$. Under this energy allocation, the (TRIGGER,TRIGGER) strategy profile is an NE of the repeated game and the total expected payoff for both source nodes can be calculated as $\Pi_{1}=0$ and $\Pi_{2}=\mathrm{E}\left\{\mathcal{E}_{2}^{*}[n]\right\}$. This energy allocation, however, is likely to be inefficient in the sense that there may be other energy allocations that result in a better total expected payoff for one or both source nodes. The question of how to select an efficient and mutually agreeable energy allocation is considered in the following section.

\section{EfFicient Cooperative Energy Allocation}

When the set $\hat{\mathcal{U}} \backslash(0,0)$ is not empty, selfish nodes will attempt to arrive at a unique mutually agreeable payoff pair (and, consequently, a unique energy allocation) through "bargaining". The bargaining problem is one of the paradigms of cooperative game theory in which a group of two or more participants are faced with a set of feasible outcomes, any of which can be the bargaining solution if agreed to unanimously. Our use of the term bargaining here is somewhat misleading in the sense that the nodes do not actually bargain 
by communicating offers and counteroffers to each other. Rather, since the channel state is known to both source nodes and each node knows how the other will bargain, each node can determine the bargaining solution locally without any additional communication. The technique of uniquely dividing a surplus among selfish players is commonly called "bargaining" in the cooperative game-theory literature, however, and we will use this term here for consistency.

Let us define the pair $(\mathcal{U}, \Delta)$ as the bargaining problem, where $\mathcal{U}$ is the set of all feasible stage-game payoff pairs and $\Delta=(0,0)$ is the disagreement payoff. If both sources fail to reach an agreement, they use direct transmission to deliver their packets to their intended destinations and receive the disagreement payoff in the current stage game. Note that $\Delta$ is always in $\mathcal{U}$ because direct transmission is always feasible. It is assumed that $\mathcal{U}$ is a convex and closed set, bounded from above. Given the definition of the bargaining problem and the set of Pareto-efficient payoff pairs $\hat{\mathcal{U}}$, an axiomatic bargaining solution is a function $B$, based on a set of "reasonable" axioms, that maps every $(\mathcal{U}, \Delta)$ to a unique member of $\hat{\mathcal{U}}$. Specifying these axioms serves to characterize the solution uniquely from among the set of Pareto-efficient points.

The most commonly used axiomatic bargaining solution is the Nash Bargaining Solution (NBS) which is based on four simple and well-accepted axioms and has been shown to have close connections to subgame-perfect equilibria in infinite horizon games [14]. These axioms can be briefly described as

1) (Pareto-efficiency) the bargaining solution $B(\mathcal{U}, \Delta)$ must be Pareto-efficient

2) (independence of linear transformations) if $T=$ $a x+b$ with $a>0$, then the bargaining solution $B(T(\mathcal{U}), T(\Delta))=T(B(\mathcal{U}, \Delta))$, i.e. the bargaining solution must be independent of the utility scales of the players

3) (symmetry) the bargaining solution $B(\mathcal{U}, \Delta)$ will give equal payoffs to both players if the set $\mathcal{U}$ is symmetric in the sense that $\left(u_{1}, u_{2}\right) \in \mathcal{U}$ implies $\left(u_{2}, u_{1}\right) \in \mathcal{U}$

4) (independence of irrelevant alternatives) if $\mathcal{V} \subseteq \mathcal{U}$ and $B(\mathcal{U}, \Delta) \in \mathcal{V}$, then $B(\mathcal{V}, \Delta)=B(\mathcal{U}, \Delta)$, i.e. the addition of irrelevant alternatives does not affect the bargaining solution

Other axiomatic bargaining solutions based on different sets of axioms include the Raiffa (Kalai-Smorodinsky) [16] (RBS) and the Modified Thomson (MTBS) bargaining solutions. A unified view of all these axiomatic models is presented in [17].

The NBS, RBS, and MTBS bargaining solutions can all be expressed as

$$
B_{\beta}(\mathcal{U}, \Delta)=\arg \max _{\left(w_{1}, w_{2}\right) \in \hat{\mathcal{U}}} w_{1} w_{2}
$$

where $\beta$ is a scalar parameter specified by the bargaining solution $(\beta=0$ for NBS, $\beta=1$ for RBS, and $\beta=-1$ for MTBS) and

$$
w_{i}:=\frac{\pi_{i}}{m_{i}}+\beta\left(1-\frac{\pi_{j}}{m_{j}}\right)
$$

is the preference function of source node $i$, with $j \in\{1,2\}$, $j \neq i$, and $m_{i}$ is the maximum stage-game payoff of source $i$ over $\mathcal{U}$. The value of $\beta$ in the preference function implies a tradeoff between a player's own gain and the other player's losses, normalized by each player's maximum gain. When players use the NBS $(\beta=0)$, the bargaining solution is such that players only maximize their own payoff without consideration of the losses incurred by the other player. When players use the RBS $(\beta=1)$, the bargaining solution is such that each player's payoff is proportional to its maximum. Finally, when players use the MTBS $(\beta=-1)$, each player has the same preference function and the bargaining solution is such that the sum of the normalized payoffs $\pi_{1} / m_{1}+\pi_{2} / m_{2}$ is maximized.

To illustrate the feasible payoff set, the Pareto-efficient subset, as well as the different bargaining solutions, Figure 3 shows the positive quadrant of the feasible stage-game payoffs as well as the NBS, RBS, and MTBS for a two-player relaying game using orthogonal amplify-and-forward (OAF) cooperation with channel state $H_{12}=H_{21}=5, H_{13}=0.5$, $H_{14}=5, H_{23}=3$, and $H_{24}=0.9$ and an SNR=10dB QoS constraint. The maximization in (1) is performed numerically for $\beta \in\{-1,0,1\}$ to obtain the three different bargaining solutions. The centrally controlled maximum total payoff (or, equivalently, minimum total energy), is also plotted for comparison.

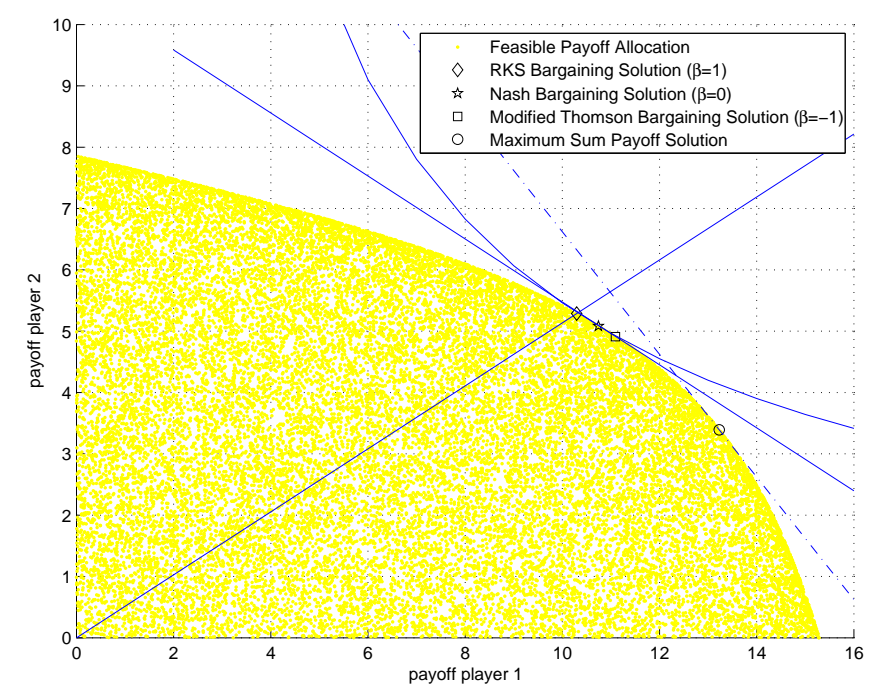

Fig. 3. Illustration of NBS, RBS, and MTBS two-player bargaining solutions over the set of feasible stage-game payoffs, $\mathcal{U}$.

As a final comment on the use of bargaining solutions to find an efficient resource allocation to which both selfish sources will agree, it should be reiterated any bargaining solution will imply an energy allocation that must satisfy the NE criteria developed in Proposition 1 (or Lemma 1 in the case of nonfading channels). If the bargaining solution implies an energy allocation that does not satisfy the NE criteria, both nodes will know this and will use direct transmission to avoid defection and triggering punishment. 


\section{V. $K>2$ Player Relaying Game}

In the case when there are $K>2$ source nodes in a given transmission session, we restrict our attention to the particular scenario in which the source nodes form fixed twoplayer partnerships for the duration of a transmission session. The central problem is then the assignment of partners to each source node (except one, when $K$ is odd). Specifically, we consider the problem of how to form stable partnerships endogenously by selfish source nodes.

We define a pairing instance $\mathcal{P}$ as a set of two-player partnerships in which all but at most one source nodes are disjointly paired. It is not difficult to show that each pairing instance in a $K>2$ player relaying game is an equilibrium with respect to unilateral deviations when the energy allocations are determined by a bargaining solution and the NE conditions are satisfied. Pairing instances may not be an equilibrium with respect to multi-player deviation, however, where two or more players leave their current partners and form different partnerships. As an example, consider a network with $K=4$ source nodes denoted as $\mathcal{S}=\{1,2,3,4\}$ and a pairing instance $\mathcal{P}=\{\{1,2\},\{3,4\}\}$. Suppose that all nodes receive an identical expected payoff of $\pi_{\mathcal{P}}>0$ under this pairing instance. Suppose further that, under pairing instance $\mathcal{Q}=\{\{1,3\},\{2,4\}\}$, nodes 1 and 3 each receive a payoff of $\pi_{\mathcal{Q}}>\pi_{\mathcal{P}}$ while nodes 2 and 4 receive a payoff of zero. It is clear that nodes 1 and 2 both improve their payoff by deviating from pairing instance $\mathcal{P}$ to $\mathcal{Q}$, and they can do so without any consent (or repercussions) from nodes 3 and 4. Hence, although pairing instance $\mathcal{P}$ is an equilibrium with respect to unilateral deviation, it is not an equilibrium with respect to multi-player deviations.

While there are many notions of equilibrium in $K>2$ player games, we restrict our attention here to the notion of a pairwise-stable network [19]. A pairwise-stable network is a pairing instance that is immune to any improving two-player deviations, where an improving two-player deviation in our context is a deviation in which two players sever their current partnerships and form a new partnership such that at least one player in the new partnership receives a strictly greater expected payoff while the other player in the new partnership receives an expected payoff no worse than before. The pairing instance $\mathcal{P}$ in the previous paragraph is clearly not a pairwise stable.

The problem of how to endogenously form a pairwise-stable network among selfish nodes has been studied extensively under the title of stable matching problems [11]. Stable matching problems are generally divided into two categories: two-sided matching and one-sided matching. In a two-sided matching problem, also referred to as the marriage problem, there are two sets of participants and the matching is a oneto-one mapping between the two sets. This is the classic matching problem discussed in [11], where it is shown that every instance of the two-sided matching problem always admits at least one stable solution. In the one-sided matching problem, a matching results in a partition of the single set of participants into disjoint pairs. This is a generalization of the marriage problem and is known as the roommate problem. A major difference between a two-sided (marriage) problem and a one-sided (roommate) problem is that the roommate problem may not necessarily have a stable matching.

In the context of a $K>2$ player relaying game, the matching problem is one-sided since the source nodes are homogeneous. In the absence of central control, the source nodes can endogenously attempt to form a stable matching by first computing the bargaining payoffs (and checking the NE conditions) for each of the $K-1$ possible partners in the network. These payoffs then imply a preference table, known to each node, that is used as the input to the Stable Roommate (SR) algorithm [12] computed locally at each node to determine a pairwise-stable matching, if one exists. If a pairwise-stable matching exists, the nodes then cooperate in the transmission session with these pairings using transmission/relaying energies specified by the appropriate bargaining solution (or direct transmission if the bargaining solution does not satisfy the NE conditions). If a pair-wise stable matching does not exist, one approach is to resort to direct transmission in the current transmission session. Another approach is to locally compute the centrally controlled pairings and form partnerships based on these pairing. Both of these approaches are demonstrated in the numerical results in the following section.

\section{Numerical Results}

To demonstrate the energy efficiency of wireless networks with selfish energy allocation and selfish partner selection, this section provides a numerical example for a wireless network with non-fading path-loss channels using orthogonal amplify-and-forward (OAF) cooperative relaying. In each transmission session, $K$ source and $K$ destination nodes are randomly placed on a disk of radius $R=10$ meters (with uniform distribution). The squared channel magnitude between each node pair $i$ and $j$ is then calculated as $H_{i j}=$ $\left(\left(x_{i}-x_{j}\right)^{2}+\left(y_{i}-y_{j}\right)^{2}\right)^{-\gamma / 2}$ where $\gamma=4$ is the pathloss parameter and $\left(x_{i}, y_{i}\right)$ is the cartesian coordinate pair of node $i$. The relative energy efficiency of several schemes, averaged over the random node positions, are compared in Figure 4 with respect to a system with centrally controlled (CC) energy allocations and $\mathrm{CC}$ pairing assignments. In Figure 4, a relative energy efficiency of one corresponds to the minimum total network transmission energy (obtained through CC energy allocations and $\mathrm{CC}$ pairing assignments) and a relative energy efficiency of zero corresponds to direct transmission.

The results in Figure 4 show that a system with purely selfish energy allocations (NBS/NE) and endogenously formed node pairings (SR) can achieve a relative energy efficiency of approximately half of that of a system with centrally controlled energy allocations and pairings for values of $K \geq 10$. Since a stable roommate node pairing solution does not always exist, we provide "optimistic" and "pessimistic" bounds on the relative energy efficiency of NBS/NE energy allocations with 
SR pairings by using the centrally controlled pairing assignment and direct transmission, respectively, when a SR pairing solution is not found. Note that the results corresponding to $\mathrm{CC}$ energy allocations are not stable with respect to unilateral deviations in the sense of an NE. This is because some nodes may receive negative payoffs under CC energy allocation and these nodes would rationally choose defection. Also note that all of the results corresponding to NBS/NE energy allocations are stable with respect to unilateral deviations in the sense that no single node can improve its payoff by defecting. The NBS/NE results with SR pairings are stable with respect to bilateral (pairwise) deviations when the SR pairing solution exists, whereas $\mathrm{CC}$ and random pairings are not, in general, stable with respect to bilateral deviations.

This numerical example confirms that, in the absence of central control in a wireless ad hoc network, sources can endogenously form cooperative pairs and selfishly allocate their transmission/relaying energies to improve the overall network energy efficiency with respect to direct transmission. The results also suggest that SR pairings are significantly more efficient than random pairings and only suffer a small loss with respect to CC pairings.

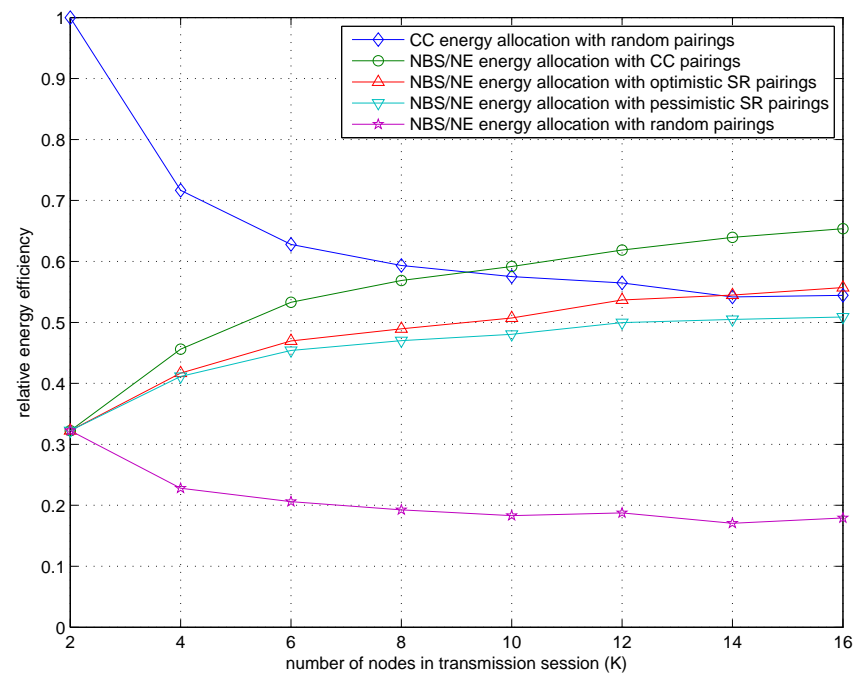

Fig. 4. Relative energy efficiency of a wireless network using OAF cooperative relaying with centrally controlled (CC) or selfish (NBS/NE) energy allocation and with random, centrally controlled (CC), or optimistic/pessimistic stable roommates (SR) partner assignments.

\section{CONCLUSION}

This paper employs both non-cooperative and cooperative game theoretic tools to analyze the energy efficiency of wireless ad hoc networks with selfish energy allocation and endogenous partner selection. The novelty of this study is that cooperation is established without the added complexity of extrinsic incentive mechanisms, altruistic nodes, and/or community enforcement. We first described a twoplayer repeated relaying game and developed the necessary and sufficient conditions under which "natural" cooperation is possible. These conditions were derived for both fading and non-fading channels. By incorporating axiomatic bargaining models, we then showed how to calculate a unique Paretoefficient cooperative energy allocation that can be locally computed and agreed upon by selfish nodes without central authority. We then extended the two-player model to networks with $K>2$ players and proposed a technique to endogenously form cooperative partnerships without central control through the stable roommate algorithm. Finally, we provided numerical results based on OAF relaying and quantified the energy efficiency of an ad hoc wireless network with selfish sources with respect to a network with centrally optimized energy allocations and pairings.

\section{REFERENCES}

[1] Q. Zhao and L. Tong, "Energy efficiency of large-scale wireless networks: Pro-active vs. reactive networking," IEEE Journal on Selected Areas of Communications, vol. 23, pp. 1100-1112, May 2005.

[2] S. Marti, T. J. Giuli, K. Lai, and M. Baker, "Mitigating routing misbehavior in mobile ad hoc networks," in in Proc. ACM/IEEE Annual International Conference on Mobile Computing and Networking (Mobicom), pp. 255-265, 2000

[3] J. Yang, A. G. Klein, and D. Brown III, "Cooperation without extrinsic incentive mechanisms in wireless networks with selfish nodes," Accepted to appear in IEEE Signal Processing Magazine, 2009.

[4] J.-P. Hubaux, T. Gross, J.-Y. Le Boudec, and M. Vetterli, "Towards self-organized mobile ad hoc networks: the terminodes project," IEEE Comm. Mag., vol. 39, pp. 118-124, Jan. 2001.

[5] L. Blazevic, L. Buttyan, S. Capkun, S. Giordano, J.-P. Hubaux, and J.-Y. L. Boudec, "Self organization in mobile ad hoc networks: the approach of terminodes," IEEE Communications Magazine, vol. 39, pp. 166-174, June 2001.

[6] M. Jakobsson, J.-P. Hubaux, and L. Buttyan, "A micro-payment scheme encouraging collaboration in multi-hop cellular networks," in Financial Crypto 2003, 2003.

[7] L. Lai and H. El Gamal, "On cooperation in energy efficient wireless networks: The role of altruistic nodes," IEEE Transactions on Wireless Communications, vol. 7, pp. 1868-1878, May 2008.

[8] M. Felegyhazi, J.-P. Hubaux, and L. Buttyan, "Nash equilibria of packet forwarding strategies in wireless ad hoc networks," in IEEE Trans. Mobile Computing, Vol. 5, May. 2006, pp. 1044-1049, May 2006.

[9] J. Yang and D.R. Brown III, "Energy efficient relaying games in cooperative wireless transmission systems," Signals, Systems and Computers, 2007. ACSSC 2007. Conference Record of the Forty-First Asilomar Conference on, pp. 835-839, Nov. 2007.

[10] J. N. Laneman, D. N. C. Tse, and G. W. Wornell, "Cooperative diversity in wireless networks: Efficient protocols and outage behavior," IEEE Transactions on Information Theory, vol. 50, no. 12, pp. 3062-3080, 2004.

[11] D. Gale and L. Shapley, "College admissions and the stability of marriage," The American Mathematical Monthly, vol. 69, pp. 9-15, January 1962.

[12] D. Gusfield and R. W. Irving, The Stable Marriage Problem: Structure and Algorithms. Cambridge, Massachusettes: The MIT Press, 1989.

[13] J. Yang, D. Gündüz, D.R. Brown III, and E. Erkip, "Resource allocation for cooperative relaying," in Proceedings of the Conference of Information Sciences and Systems (CISS 2008), (Princeton, NJ), Mar 2008.

[14] K. Binmore, Fun and Games: A Text on Game Theory. Lexington, MA: D.C. Heath, 1992.

[15] R. Axelrod, The Evolution of Cooperation. Basic Books, 1984.

[16] E. Kalai and M. Smorodinsky, "Other solutions to nash's bargaining problem," Econometrica, vol. 43, no. 3, pp. 513-518, 1975.

[17] X. Cao, "Preference functions and bargaining solutions," in IEEE Conference on Decision and Control, pp. 164-171, 1982.

[18] J. Yang and D.R. Brown III, "The effect of channel state information on optimum energy allocation and energy efficiency of cooperative wireless transmission systems," in Proceedings of the Conference of Information Sciences and Systems (CISS 2006), (Princeton, NJ), pp. 1044-1049, Mar 2006.

[19] M. Jackson and A. Wolinsky, "A strategic model of social and economic networks," Journal of Economic Theory, vol. 71, pp. 44-74, June 1996 